\title{
L'education de la fille dans la famille Algerienne traditionnelle
}

\section{Dr .Rajia BEN ALI \\ Université de Batna, Algérie}

\section{Résumé :}

Dans le cadre de la problématique du changement social en Algérie, l'éducation familiale nous a paru être un sujet très important, elle est même le centre de la confirmation de ce changement et de la rupture avec le modèle traditionnelle. Par cet article nous voulons aborder quelques détailles de l'éducation de la fille dans la famille algérienne traditionnelle. Une éducation basée sur la reproduction du système social existant, et qui transmis aux filles comme aux garçons des rôles et des statuts bien définis, qu'ils ne doivent pas transgresser. La famille traditionnelle, à travers l'éducation, inculque à la fille, dés sa naissance, des qualités, des valeurs et des comportements complètement différents de ceux du garçon, comme nous allons le montrer.

الملخص:

تعد التربية الأسرية، في اطار اشكالية التغير الاجتماعي في الجزائر،

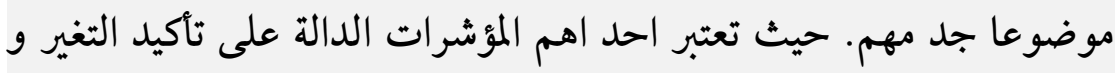

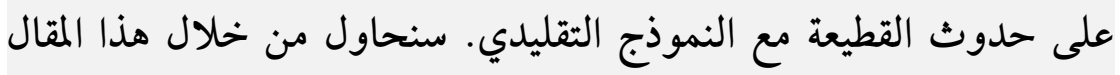

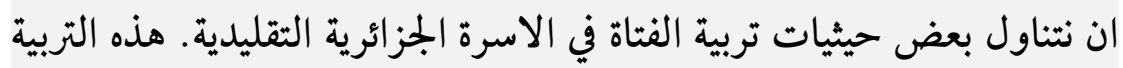

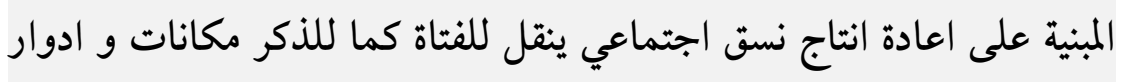

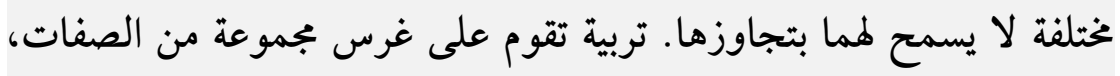

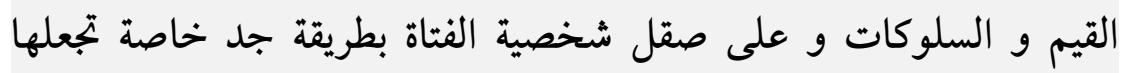
تتوافق و متطلبات و خصائص المجتمع الابوسي القائم في الفترة التي تلت الت الاستقلال كما سنعرضه فيما يلي. 


\section{Introduction :}

Parler ou décrire l'éducation de la fille au sein de la famille algérienne traditionnelle peut paraitre un sujet peu intéressent, du fait des différents changements qu'a subit la société algérienne. Aujourd'hui, la vie familiale a beaucoup changé et la société a évolué vers plus de mixité, à commencer par l'école qui est obligatoire pour les deux sexes et au sein de laquelle les garçons et les filles sont contraints à occuper le même espace et à recevoir la même instruction. Mais puisqu'il s'agit de changements, la démarche classique est de se doter d'un repère par rapport auquel nous pouvons appréhender la différence, d'où l'importance de cet article.

En outre, nous voulons mentionner ce que nous essayerons de décrire et d'analyser ci-dessous pourra paraître caricatural, excessif pour certains traits mais c'était l'éducation conçue aux filles et mise en œuvre dans la famille algérienne des années soixante, et qui persiste pour certains pratiques jusqu'à nos jours. Cette éducation s'appliquait en ville comme à la campagne, elle peut parfois sur certains traits ou dans certaines régions être différente de l'image retracée, mais les écarts ne sont pas très importants.

\section{1- La naissance d'une fille :}

La naissance d'une fille dans la famille algérienne traditionnelle est ressentie avec moins de joie par les parents que celle du garçon. Si la naissance de ce dernier s'accompagne de 
youyous et de fêtes, celle de sa sœur se fait dans le silence. Le sexe de la fille est assimilé, selon les régions d'Algérie par des qualificatifs peu flatteurs, voire vexatoires de :'citrouille" à Constantine, "cloporte' à Saïda, "navet" à Tlemcen, " ténèbres", dans le Sétifois où il est dit que les ténèbres ont envahi la maison.

$\mathrm{Au}$ fait, son accueil dépend de son rang de naissance et du nombre de garçons dans sa famille. Si elle est la première ou si elle a des frères en nombre jugé suffisant, sa naissance sera mieux acceptée, sans que la famille manifeste aucun signe de joie. Autrement, la mère doit supporter en silence la commisération des proches, le ressentiment et l'hostilité du mari et des beaux-parents, l'humiliation de s'entendre renvoyer l'impuissance à engendrer des enfants mâles.

Nombreuses sont les mères qui ont vécu avec souffrance, culpabilité, mépris envers elles-mêmes, envie pour les autres plus « chanceuses » l'absurde drame de ne réussir à mettre au monde que des filles. Il n'est pas rare de voir des femmes enchaîner grossesse après grossesse dans l'espoir d'avoir un garçon.

L'attente de l'heureux événement par le père se fait dans l'angoisse ; s'il n'entend pas les youyous saluant la naissance de son bébé il doit s'attendre à des consolations de la part de son entourage. Surtout s'il n'a pas du tout de garçon et que cette dernière naissance vient après celle d'autres filles. Un sentiment de honte l'envahit et il n'est pas étonnant de le voir se cacher des regards des autres. Dans 
certains cas, il arrive même qu'il répudie sa femme le jour même de l'accouchement, la rendant génétiquement responsable du sexe de l'enfant, celle qui ne peut avoir de garçons.

Bien que ces attitudes soient fortement réprimandées dans le Coran verset (16-58 et 16-59) : «Et lorsqu'on annonce à l'un d'eux une fille, il se cache des gens, à cause du malheur qu'on lui a annonce. Doit-il la garder malgré la honte, ou l'enfouira-t-il dans la terre? Comment est mauvais leur jugement !». La culture patriarcale prend le pas sur la religion sur ce point.

L'accouchement qui devrait être un événement heureux dans la vie du couple se transforme alors en une sorte de fatalité qu'il faut éviter d'évoquer. Il arrive que le père taise l'accouchement «raté » de son épouse de peur d'entendre le mot Meskine (pauvre). Les gens se lamentent ouvertement sur leur sort en utilisant des expressions telles que : "les pauvres ils n'ont pas d'enfants, ils n'ont que des filles; que Dieu leur donne de la patience،, comme si les filles n'étaient pas comptées comme des enfants. Dans la pensé patriarcale seuls les garçons comptent.

Dès l'enfance le bébé fille vit une sorte de discrimination. Si bébé elle vit une relative symbiose avec sa mère et l'entourage familial féminin comme tout petit enfant, et elle fera l'objet de soins assidus, de jeux fréquents, de berceuses, de rengaines et de mots câlins; les études ont montré que le sexe du bébé module le comportement et les attitudes de la mère. Sa relation avec ses parents 
n'est pas aussi intense que celle de son frère, comme si son sexe limitait ou freinait l'élan maternel sans parler des rapports avec le père ou l'entourage masculin.

Sylvie Garnero ${ }^{(1)}$ (1982) lors de ses observations algéroises a remarqué une différence très nette de traitement par les mères de bébés filles et par les mères de bébés garçons. Ainsi les bébés filles sont souvent plus emmaillotés que les bébés garçons. De plus on serre plus lorsqu'on emmaillote les bébés filles comme si on leur appliquait volontiers au berceau les contraintes qui les prédisposaient à la dépendance et à la soumission. Même l'allaitement est différemment donné aux bébés; il est moins fréquent et souvent $\operatorname{artificiel}^{(2)}$.

lorsqu'il s'agit des filles ; mieux encore il dure généralement moins longtemps. F. M'rabet ${ }^{(3)}$ affirme que dans le cas où la mère donne naissance à des jumeaux de sexes différents, le garçon serait toujours allaité en premier, la fillette sevrée la première. Il est aussi prouvé que les filles sont moins portées par leurs mères et facilement laissées aux autres femmes de la famille. C. Lacoste Dujardin ${ }^{(4)}$.parle d'un lien très fort entre mère et fils et beaucoup plus durable, plus étroit qu'entre mère et fille ; plus tôt sevrée et facilement confiée à d'autres femmes ou fillettes.

De même, l'éducation des fillettes, laissée à la seule responsabilité des femmes les défavorise en cas de maladie et de soins à leur apporter notamment à l'extérieur : en effet un petit 
garçon malade attire la sollicitude des hommes qui s'en soucient davantage et l'emmènent plus volontiers que la fille à la consultation médicale. Quant aux petites filles, elles sont souvent réduites aux seules ressources de soins traditionnels des femmes.

Ce n'est pas par hasard si le taux de mortalité des filles était plus élevé que celui des garçons. C. Lacoste Dujardin ${ }^{(5)}$.rapporte que dans les villages qu'elle a fréquentés et étudiés en quatre ans de 1968 à 1971, les petites filles mouraient encore deux fois plus que les petits garçons dans les premières années de leur vie : un garçon sur treize et une fille sur six. Cependant, il faut noter que cette différence dans les premières années n'est pas spécifique à la famille algérienne. E. Gianini Belotti décrit des phénomènes similaires dans son livre " Du côté des petites filles" publié en 1974 dans la sphère méditerranéenne.

\section{2- Les principes de l'éducation :}

Dans une famille qui se replie sur elle-même, qui a peu de rapport avec les individus qui n'appartiennent pas au cercle familial et où il est de principe que: "les fils de nos fils sont nos fils, les fils de nos filles sont des étrangers ", la fille est elle-même perçue comme un étranger, un passager qui ne tardera pas à repartir. Il est souvent dit lors de sa naissance : il est né un membre de plus dans la famille qui n'enrichit pas la maison, mais enrichit celle des autres. Destinée à la procréation dés qu'elle est pubère, la fille ne demeurera dans la maison parentale que pour un court moment. Elle quittera 
jeune sa famille pour passer le reste de sa vie dans une autre famille. Ce genre de perception joue sur la nature des relations entre la petite fille et ses parents. Contrairement au garçon, les parents savent dés la naissance de leur enfant que leurs relations avec elle seront peu durables, temporaires et moins fortes. Pour cette famille où elle ne vit qu'à titre provisoire, la fille représente un grand danger, elle est l'élément fragile sur lequel repose le fameux honneur familial. De même que, destinée à sortir de sa famille pour être intégrée à une autre, elle sera une sorte d'ambassadrice, de représentante de tout un lignage, qui sera jugé à travers elle. Pour cela, son éducation est estimée capitale et le peu de temps qu'elle va vivre dans sa famille sera consacré à faire du bébé qu'elle est une femme dont on garantit tous les comportements. Ne l'élève -t- on pas pour les autres?

Ainsi, l'éducation de la fille dans la famille algérienne traditionnelle, est perçue comme étant très dure, pénible. Savoir la gérer suppose beaucoup de tact, même de force. Les répercussions d'une mauvaise éducation retomberont fatalement sur toute la famille, c'est la raison pour laquelle la mère est très exigeante et plus vigilante avec sa fille qu'avec son fils. Elle doit lui adresser matin et soir des recommandations et lui ressasser à longueur de journée des conseils afin de parfaire son éducation.

Elle aura très vite une enfance différente du garçon. Son éducation sera totalement prise en charge par les femmes qui s'investissent dés sa prime enfance à lui inculquer la façon d'être une 
femme accomplie. Pour cela, elles lui inculquent que l'objectif de sa vie doit être le mariage puis la procréation. Elles lui donnent la meilleure formation possible dés la prime enfance pour qu'elle devienne une épouse et une mère rêvée. Cet apprentissage se fait volontairement à travers leurs pratiques et comportements à son égard, les discours et les paroles qu'elles lui adressent; ou inconsciemment, à travers les dictons, les contes, les proverbes et les prescriptions qui forment une base très importante dans l'éducation de la fille.

S'exerçant principalement dans la sphère domestique, l'éducation transmise -dans la famille traditionnelle- aux fillettes est en grande partie un processus d'intériorisation des comportements et des valeurs, au cours duquel, elle va acquérir peu à peu son rôle de femme. Elle s'identifie à ce monde, en imitant les gestes qu'elle voit faire, enregistrant les interdictions qui composent les conceptions familiales, participant au déroulement de tous les rites et les fêtes qui se passent à la maison. Elle partagera les soucis, les angoisses, les secrets et les rites des femmes. Ainsi, son esprit sera modelé insensiblement sur la mentalité des femmes et elle empruntera leurs réactions.

On lui inculquera aussi la soumission. Celle-ci est une vertu dans l'éducation de la fille, il s'agit d'un véritable conditionnement qui s'applique à la fille durant toute sa vie. Si la virilité est le principe de l'éducation du garçon, la soumission avec tout ce qu'elle 
implique est celui de la fille. On lui apprend la soumission comme un précepte indiscutable. Elle doit obéir aux recommandations de son père, de sa mère, de ses aînées et des hommes sans réplique. Dans certains cas elle doit être soumise à des prescriptions injustifiables, telle que celle rapporté par N, Zerdoumi(6).

" j'ai toujours été soumise à des interdits sans qu'on prenne la peine de me les expliquer. Du reste, je ne m'aventurais jamais à poser des questions : "tu oses lever la tête devant ton père? " me lançait ma mère ».

Celles qui respectent ces enseignements, on dit d'elles «Taiâ, Mardhia» ( soumise, bénie). Le plus grand éloge que la mère fasse de sa fille est de dire d'elle : ma fille a peur de son ombre, elle ne rentre ni ne sort et elle fait tout ce que je lui demande. Pour mériter ces qualificatifs, la fille doit donc adopter le modèle des femmes de son groupe (sa mère, ses tantes, ses cousines etc....) et apprendre à intérioriser et à intégrer, au plus profond d'elle-même, qu'obéir pour une fille est une qualité principale. "Obéir est pour la femme une vertu capitale et se taire une sauvegarde que toutes n'ont pas le privilège de savoir utilisen» (7).

explique N.Zerdoumi.

L'apparence que donnera la fille au sein même du monde féminin est strictement contrôlée par la mère, que ce soit par la parole ou par les gestes, il n'est pas rare de voir des mères 
discrètement corriger soit par des gestes soit par des signes d'éventuelles erreurs de comportement, de gestuelle ou de parole afin que leur fille donne une bonne image à l'extérieur. Au fait, ces mesures sont prises dans le but de préparer la fille à ce qui l'attend dans le futur une fois mariée et vivant dans un autre foyer que celui des ses parents; là où elle sera mise sous le regard critique de sa nouvelle famille, mais et surtout sous celui de sa belle-mère et ses belles sœurs, c'est là qu'elle doit prouver qu'elle est une bonne représentante de sa famille. D'ailleurs un dicton populaire un peu fataliste dit : «les maisons des gens sont pénibles : si elles ne font pas mourir, elles vous tuent à petit feu».

C'est dans cette perspective un peu pessimiste que les filles sont éduquées, dans la peur de ce qui les attend dans la maison conjugale et surtout de l'échec du mariage, ce qui pousse les mères à être très sévères et strictes avec leurs filles. Ces dernières doivent observer un code de politesse très ferme : manifester du respect et de la considération à tous les adultes ou aînés, connaître les formules de politesse convenant à chaque situation. Ne parler qu'après qu'on lui a adressé la parole. Ne pas se montrer bavarde, ni se mêler des affaires de la famille. Tenir secrètes les conversations et la vie des femmes de la maison; à table, elle doit se montrer ni gourmande, ni difficile, manger peu, ne pas être la première à se servir ni la dernière à terminer. Quand elle sert, elle doit donner la bonne et grande part d'abord aux hommes quitte à ce qu'il n'en reste pas pour elle. Une fille bien éduquée est celle qui sait se priver de nourriture, ne pas 
réclamer son dû, et qui se contente de ce qu'on lui donne. Toute expression d'une opinion ou d'une conduite sortant de la mesure conformiste est mal venue. Faire adopter à la fille un comportement fait de réserve, de retenue et de décence est l'objectif principal. Cette première éducation est un apprentissage quasi spontané qui aboutit à inculquer à la fille les règles de bienséance et de savoir-vivre qui constituent la base essentielle de son éducation.

Autre point dans son éducation différent de celle de son frère est la ségrégation. Dés l'âge de six ans, la fille ne doit pas jouer avec les garçons, ni demeurer en présence des hommes, elle doit se conduire pendant leur présence avec prudence et respect et surtout éviter de parler aux étrangers et de fréquenter tout milieu où se trouvent les hommes, que ce soit dans la maison, dans la rue ou ailleurs. Si le garçon grandit dans le monde des femmes, il ne sera obligé de respecter la ségrégation qu'après l'âge de sa préadolescence.

La fille apprend dés sa petite enfance à subir une double ségrégation : de l'âge et du sexe. Si elle a un frère aîné elle doit subir sa jalousie et endurer ses sévices, ses caprices, ses exigences et ses gâteries. Si elle a des frères plus jeunes, elle ne tardera pas à en être la gardienne si toutefois on ne lui en laisse pas la charge complète. Encouragée à accomplir un rôle maternel dés son jeune âge, elle doit être docile, patiente et endurante avec ses frères. Du coup, ils se montrent, comme le souligne Germaine Tillon, souvent comme des 
petits despotes avec elle. Elle doit supporter leurs coups et trouver cela naturel. Pourquoi pas si les parents eux-mêmes acceptent ce genre d'attitude et vont même à encourager leur fils. Ce comportement des parents, dans la famille traditionnelle, envers les filles obéit à la crainte de la désobéissance qu'elles peuvent manifester lorsqu'elles seront adultes. Une fille doit à tout prix grandir dans la crainte révérencielle de l'homme quel que soit son statut et son âge.

Ainsi, la fille prend conscience de la supériorité de l'homme, y compris son propre père qui sera toujours absent, par pudeur ou par décence et qui freinera ses élans de tendresse envers elle, même s'il en éprouve le besoin. La ségrégation lui sera imposée avec fermeté. La fille doit être consciente de l'autorité et de la supériorité des hommes, de même qu'elle doit très tôt savoir que son monde à elle et celui des hommes, ne se rencontrent jamais. Même avec son père puisque toute communication avec lui est médiatisée par la mère.

Telles sont en général les qualités morales sur lesquelles repose l'éducation de la fille. Toutefois il reste deux autres éléments considérés aussi comme très importants: d'une part l'éducation ménagère et l'initiation à la vie de femme au foyer puisque l'idéal de la mère est de faire de sa fille une très bonne femme au foyer; et d'autre part la préservation de sa virginité, incontournable preuve de sa bonne éducation, qui lui ouvre les portes du mariage et atteste qu'elle est une femme accomplie. 


\section{2-1 - L'éducation ménagère et les devoirs}

On a vu que le statut idéal que la culture patriarcale offre à la fille est celui d'épouse et de mère. Ce statut est l'objectif de toute la famille avant d'être celui de la fille. Pour l'acquérir, la fille doit dés son jeune âge savoir maîtriser toutes les activités qu'une femme est censée faire. En plus des qualités citées dans la partie précédente, l'éducation de la fille sera dans une grande partie fondée sur l'apprentissage des tâches ménagères qui forme un des critères les plus marquants dans son éducation. Vers la sixième année et même plus tôt, son quotidien sera partagé entre le jeu et l'initiation aux travaux ménagers ce qui est affirmé par N. Zerdoumi ${ }^{(8)}$.

qui dit: "vers l'âge de six ans, la petite algérienne est à la cuisine et au balai ».

Le partage rigide des rôles dans le groupe familial, contraint la fillette à connaître très tôt tous les travaux qui concernent l'intérieur de la maison : balayer, faire la vaisselle, quérir l'eau, faire la lessive, le ménage etc... Et plus tard, l'âge avançant, elle apprendra à faire la cuisine, à coudre et à tisser. En dehors des tâches ménagères, elle doit aussi s'occuper et prendre soin de ses frères et sœurs cadets, les bercer, les garder, les faire manger et dans certains cas en prendre la responsabilité totale afin de ménager la mère, surtout si cette dernière est accablée par ses maternités successives. 
L'initiation des filles aux travaux domestiques est une préoccupation constante dans la démarche éducative de toutes les mères. Elle est présente chez elles sous forme d'inquiétude et de culpabilité. Il n'est pas rare de les voir obliger leurs petites filles, vers l'âge de douze ans, à accomplir avec résignation les durs travaux ménagers auxquels elles sont astreintes, ou leur laisser la responsabilité de toute la maison lorsque -pour des raisons économiques- elles aident leurs maris dans les travaux des champs ou le tissage des tapis ou lorsqu'elles sont malades. La fille s'habitue à ce rôle et trouvera cela naturel, surtout si elle est l'aînée. Certes, si elle a d'autres sœurs plus jeunes, elles partageront le travail ; mais tout ce qui est pénible ou demande un savoir-faire, c'est elle qui doit l'accomplir remplaçant ainsi la mère malgré son jeune âge.

Pour ce coup de main exigé d'elle, elle ne sera pas complimentée ni remerciée, au contraire elle doit savoir gré à sa mère de lui avoir enseigné son métier de femme. Puisque, plus que pour aider sa mère, cet apprentissage a pour but de décrocher un brillant mariage. Destinée à peupler la maison des autres « T’ammar dâr en-nas », celle qui saura accomplir ces tâches dans tous les détails sera la plus demandée en mariage. On entendra dire d'elle «Fahla » et « Chatra » sachant tous faire, débrouillarde. Sa maîtrise des tâches ménagères et son bon comportement seront complimentés dans les réunions féminines. On parlera d'elle et c'est de cette façon que s'ouvriront les portes des demandes au mariage, suprême 
couronnement de tous les efforts de la mère et de sa fille dans la famille traditionnelle.

Les hommes n'intervient pas sur ce plan, ils passent la majorité de leur temps en dehors de la maison et généralement ce n'est pas à eux de juger les mérites de la fille. Ce sont les femmes qui terrorisent les femmes (les jeunes filles), ce sont elles qui leur imposent d'être compétentes dans ce genre d'apprentissage. C'est la future belle mère et les belles sœurs avec qui la fille vivra une fois mariée qui vont juger son assiduité et son savoir faire.

La fille entendra dire de sa mère pendant toute sa période d'apprentissage la même phrase : «les femmes vont rire de toi » et rien que cette phrase motive ou pousse les petites filles à rivaliser entre elles et à s'améliorer de jour en jour en montrant leur savoirfaire ou leur maîtrise aux plus âgées allant parfois jusqu'à supplanter leurs aînées. Cet apprentissage, contraignant pour une enfant, est dû principalement à la précocité de l'âge du mariage. Les étapes de la vie de la petite fille se succèdent très vite et elle peut se trouver bonne à marier dés qu'elle dépasse ses dix, douze ans. F. Fanon ${ }^{(9)}$.

disait que: «la vie d'une femme algérienne ne se développe pas selon les trois temps connus en occident: enfance- pubertémariage ; la jeune fille algérienne ne connaît que deux étapes: enfance- puberté, et mariage». Mariée souvent dés sa puberté la fillette n'aura pas d'autre temps pour apprendre les tâches ménagères que celui de son enfance. Si par hasard, elle se trouve un jour, sans 
avoir eu cette chance d'apprendre son métier de femme, si elle se montre incompétente, dépensière, désordonnée ou frivole, le mari et surtout la belle-mère auront des occasions de comparaison et risqueront de s'en offusquer et le ménage en subira les conséquences, s'il ne finit pas par un divorce. Il n'est pas surprenant, ni rare d'entendre dire qu'une fille est répudiée par sa belle-mère ou son beau-père parce qu'elle n'a pas su par exemple préparer tel plat, même si elle a de très bonnes affinités avec son mari.

D'ailleurs on teste son assiduité pour les tâches ménagères juste après le jour de son mariage. Dans les Aurès par exemple, il n y a pas longtemps, la mariée se trouvait contrainte à quérir l'eau de la fontaine sur son dos, à tisser un petit tapis, voire juste le commencer, pour montrer son savoir-faire, et à préparer un certain plat traditionnel de pâtes nommé (Chekhchoukha) qui n'est pas facile à faire. Celle qui n'a pas réussi son test sera le lendemain la risée de toute sa belle-famille.

Dans la famille traditionnelle, l'intériorisation de l'idée du mariage comme objectif pour la fille et la réputation qu'aura une mère qui réussit à marier sa fille après avoir fait d'elle une bonne femme au foyer créent une grande complicité entre la mère et la fille par rapport à l'apprentissage des tâches ménagères. Cette complicité on la trouve aussi dans le souci de la mère de collecter tout ce qu'elle peut : des tapis, des parfums, des coupons de tissus, etc...pour sa fille et sa précipitation à lui inculquer le métier du tissage et de la 
couture dans la perspective de bien préparer son trousseau. Un dicton populaire dit : « dès que la petite commence à marcher regarde ce que sa mère a caché». Le trousseau (Choura) est dans sa plus grande part constitué de travaux manuels afin de montrer à la famille du futur époux les qualités, l'expérience, et le savoir-faire de la fille. La mère, en exhibant avec fierté le trousseau de sa fille dit que «chaque doigt de ma fille est doté d'un métier» vantant ainsi les différentes qualités de sa fille.

L'apprentissage des travaux domestiques est un grand pas vers la réalisation de l'objectif de la fille et de sa mère. Celle qui sous-estime cet apprentissage risque de voir sa fille rester toujours à ses cotés.

Dans ce domaine une très grande différence de traitement est appliquée aux filles et aux garçons. L'initiation aux tâches ménagères est une option exclusivement féminine. Le garçon passe toute sa tendre enfance sans subir les contraintes de ce genre d'apprentissage. Les tâches ménagères ne sont en aucun cas une affaire d'hommes ; sa moindre participation est considérée comme (Aib) tabou. Il n'est pas concevable qu'un homme s'abaisse aux travaux domestiques. Si par hasard il aide sa sœur ou sa mère dans certaines tâches ou même s'il les accomplit pour lui même, il risque d'être traité de (Mriya) femmelette et sévèrement remis à sa place et réprimandé. Par contre il apprend dès l'enfance à commander ses sœurs et à exiger qu'elles soient à son service. 


\section{2-2 - La préservation de la virginité}

Dans la société traditionnelle, l'environnement social est harmonieux. L'initiation de la fille accomplie par la mère et les autres femmes de la maison, est approfondie par le biais de tout l'entourage. Les proches, les voisins, les gens de la rue et toute la société approuvent la même idéologie quant à l'éducation de la fille. Dans ce contexte la préservation de la virginité est un des critères les plus rigides de l'éducation; elle représente un fait social institutionnel qui, est à la fois culturel, religieux et idéologique. Elle n'est pas propre uniquement à la famille algérienne puisqu'elle concerne toutes les communautés arabo-musulmanes, certaines zones de la Méditerranée et plusieurs communautés asiatiques. Le Coran (III.42, XXI.91) et la Bible (10).

(Mathieu 25,1-13) en parlent aussi. Quoi qu'il en soit, elle est pour la famille algérienne un indice très important de la bonne éducation, l'emblème de la chasteté morale et physique de la jeune fille et la pierre de touche de la morale de toute sa famille.

La jeune fille vierge est extrêmement valorisée dans le discours religieux qui contribue à faire d'elle le prototype de la beauté féminine. D'ailleurs c'est une des qualités des houris du paradis promis pour les bons musulmans. Par contre, une fille non vierge et qui s'aventure dans des relations hors mariage est identifiée à la Fitna. F. MERNISSI (11). 
explique qu'elle est plus que la Fitna, et qu'elle est Satan luimême. Cette ressemblance se trouve dans son invitation à faire le mal et à détruire l'ordre social. En Islam, la virginité est associée à la pureté. Pureté de l'âme, de la personne, de la famille, de la lignée et de toute la société. Ainsi, elle doit être l'objectif de tout musulman.

En outre, elle est dans la conception populaire une règle morale très importante qui représente une des bases sur lesquelles repose l'idéologie de la famille algérienne. Elle symbolise le côté pratique et le seul indice concret de l'honneur. De ce fait, la préservation de la virginité ouvre grand les portes du mariage à la jeune fille et signe rituellement son entrée dans le monde de «la socialité sexuelle » (12). C'est pour cela qu'elle représente un aspect principal de son éducation qui nécessite la mobilisation de toute la famille: femmes, hommes, jeunes, adultes tentent par tous les moyens de préserver intact un hymen qui une fois perdu ne pourra jamais être substitué du fait qu'il figure la dignité et l'honorabilité de tout le clan familial.

Avant le mariage, la sexualité et la virginité de la jeune fille sont aperçues comme un héritage collectif qu'il faut placer sous haut contrôle et qui ne sauraient en aucun cas être laissés à la gestion des individus, comme ils ne sauraient être liés à l'affectivité. C'est pourquoi une fois que la fillette dépasse l'âge de six ans, la sauvegarde de sa virginité devient une sorte d'obsession collective et un des rôles fondamentaux qui implique en plus d'elle et de sa mère 
tous les hommes de la famille. Le père, les frères, les oncles et les cousins se trouvent contraints de contrôler et d'exercer une autorité absolue sur les femmes mais surtout sur les filles non mariées (vierges) de la famille.

Dans la culture patriarcale, le courage, l'endurance et l'estime des obligations des hommes ne sont valorisés que par la bonne conduite morale des femmes de la maison et surtout par la garantie de la virginité des filles. Un homme qui maîtrise, contrôle et surveille le comportement sexuel de sa sœur ou de sa fille peut tenir honorablement sa place dans son groupe familial, voire dans tout le village, c'est ce qui garantit sa virilité. Il existe comme une véritable hantise de perdre son honneur surtout dans une société où le droit de regard de l'extérieur sur les individus et les entités familiales est très courant.

Dans une société où rien n'est plus important que la conformité de la jeune fille à la normativité de son groupe, la simple violation nécessite l'intervention des hommes de la famille qui doivent défendre leur honneur, et ne jamais laisser rabaisser leur prestige (En nif). Le Nif ce qui en traduction littérale signifie le (nez), l'organe physique le plus apparent extérieurement, veut dire aussi pour les Algériens, le prestige et l'estime de soi. P. Bourdieu (13)

le définit comme étant avant tout ce qui porte à défendre à n'importe quel prix une certaine image de soi destinée aux autres. 
Cette image est celle de tout le groupe, elle peut s'étendre même dans le temps. Perdre la virginité est une tare qui rejaillit sur toute la famille, et par foi sur plusieurs générations. Pour les membres de la famille traditionnelle l'honneur est indivis, il n'est pas un sentiment individuel. Il comprend aussi bien la défense de l'intimité (Horma) du groupe restreint que celle du groupe élargi.

En se défendant par le Nif c'est l'ensemble de tout le groupe familial qui est défendu. Mieux encore, respecter l'honneur et l'intimité d'autrui c'est travailler, dans un système d'échanges symboliques, au respect de la sienne propre. Toute transgression peut avoir des conséquences néfastes qui peuvent arriver jusqu'à la criminalité. Les histoires «d'épuration" ne sont pas rares, elles alimentent les instances des tribunaux même de nos jours.

A partir de là, la fille, dans la famille traditionnelle, est pour la famille celle qui cristallise tout le système honorifique. La préservation de sa virginité représente l'un des éléments fondamentaux de son éducation et un enjeu essentiel pour le capital symbolique familial qu'elle concrétise. La fille, qui dans le système patriarcal est perçue comme inférieure à l'homme, détient entre ses mains le destin de tous les hommes de la famille. L'honneur familial est dépendant d'elle et cela suffit pour qu'elle soit perçue comme un grand danger qui menace la famille. Ne l'assimile-t- on pas dans le langage quotidien à une bombe à retardement qui peut exploser à n'importe quel moment? 
Ainsi, la fille est perçue à la fois comme menacée et menaçante. Elle est à la fois un danger et un élément fragile qu'il faut savoir manier, mais avec beaucoup de contrôle, d'autorité, et de suspicion. La mère étant responsable aux yeux de tous de la conduite et de la réputation de sa fille, tient à lui assurer une protection particulière, une protection faite d'interdictions, de contrôle et de surveillance ; car elle éprouve malgré elle une grande inquiétude.

Il est souvent dit : "Comme est difficile la grossesse d'une fille et comme est difficile son éducation'.' De là, la mère commence par inculquer à sa fille les principes prudentiels afin qu'elle soit ouverte aux dangers qui l'entourent. Elle tisse entre elle et les hommes un voile de la honte qui ne se déchire que le jour de ses noces. Elle lui présente l'homme comme un danger qu'il faut éviter et à qui ne jamais faire confiance, 'faire confiance aux hommes c'est confier de l'eau à un tamis", (14).

Toutes ces précautions poussent la fille dés sa tendre enfance à s'écarter des garçons et à se mêler de moins en moins à eux, qu'ils soient de son âge ou plus âgés. En leur présence elle commence à se sentir confuse, elle doit les fuir et les traiter en ennemis ; pourquoi pas puisque tout ce qu'on lui a appris sur les hommes tend à lui donner le sentiment de vulnérabilité et de danger épouvantable si elle les fréquente, pour elle et pour toute sa famille.N. Zerdoumi ${ }^{(15)}$. 
rapporte un discours tenu par une mère à sa fille de neuf à dix ans en disant: "Maintenant, il faut que tu fasses attention tu es grande et les garçons vont te regarder. Tu vas avoir "un peu de seins" et cela suffit, si tu vas avec les garçons, pour risquer de perdre la rougeur du visage. Tu auras du sang sur les cuisses. C'est terrible de ne plus être vierge; tu ne pourras plus te marier car le soir de tes noces ton mari s'en apercevra et te mettra nue dans sac comme une robe sale. Il te fera ramener à la maison de ton père sur un âne. Tout le monde se moquera et l'honneur de la famille sera sali à jamais».

La rougeur du visage (Tahmirat el wajh) signifie la fierté de garder l'honneur intact. La fille algérienne doit dans toutes les circonstances posséder cette fine membrane que l'on appelle hymen. C'est peut-être la partie la plus importante de son corps. Il ne lui suffit cependant pas de la posséder, encore faut-il qu'elle soit capable de saigner, maculant ainsi visiblement le drap de sa nuit de noces, pour attester la virginité (la bonne éducation) de la jeune fille. Beaucoup de jeunes filles, non sensibilisées au problème des règles mais endoctrinées par cette phobie de la préservation de la virginité, sont terrorisées le jour de leurs premières règles croyant avoir perdu leur virginité à cause du saignement.

La mère, dans la famille traditionnelle, poursuit son rôle contraignant auprès de sa fille et au fur et à mesure que celle ci grandit, elle augmente son contrôle, sa vigilance et multiplie les 
interdits. Tous les moyens sont utilisés pour étouffer sa sexualité. Arrivant à la pré-puberté, vers les douze ans la jeune fille, surveillée continuellement, doit redoubler de discrétion dans tous ses mouvements, ses gestes et ses paroles. Elle développe aussi un étrange sentiment de honte vis-à-vis de son propre corps qu'elle ne maitrise pas : elle essaye de cacher tout ce qui en est visible et qui signifie qu'elle est devenue femme. Franz Fanon ${ }^{(16)}$.

rapportait que : «La jeune fille algérienne a honte de son corps, de ses seins, de ses menstrues. Elle a honte d'être femme devant les siens. Elle a honte de parler devant son père, de regarder son père. et son père aussi a honte devant elle... L'interdiction est ici telle, les prohibitions sont à ce point inscrites au centre même de la personnalité que la coprésence elle-même devient insupportable».

La honte «hachma» peut amener la fille jusqu'à l'effacement total. Elle est idéalisée socialement tant qu'elle se manifeste par son absence de l'espace extérieur, moins elle est visible plus on la valorise. Cette absence de l'extérieur (Hajba) et toutes ces opérations d'éducation et de personnalisation aboutissent à faire naître en elle prématurément une angoisse. L’angoisse de garder intacte sa virginité, pilier sur lequel repose toute la structure de la famille, puisque cette dernière est l'élément clé le jour de sa mise à l'épreuve, le jour où les mérites éducatifs de la famille seront évalués. 
L'angoisse du moment de vérité (nuit de noces) est portée par toute la famille, les pères intériorisent en eux une grande souffrance jusqu'au jour du mariage et de la délivrance. La séquence du rituel nuptial représente la phase terminale d'un long travail éducatif et dont le groupe social désormais élargi à la belle-famille, a besoin de connaître les résultats. On peut imaginer sans mal l'affolement qui saisit le groupe familial anxieux d'assister à l'écroulement de son capital symbolique en matière de morale et d'honorabilité sociale. F. Mrabet (17).

dit que : «Chaque père a peur comme s'il avait un ouf entre les doigts, s’il le laisse tomber il est fichu ». Laisser tomber c'est relâcher la vigilance concernant la surveillance et le contrôle de la fille, de même qu'un dicton algérien parle de la virginité de la fille en l'associant à un œuf dit que: «La fille, il faut la manier avec beaucoup de précaution comme l'cuf, s'il se casse on ne le répare pas». C. Lacoste Dujardin (18).

commente ce dicton en disant que l'œuf parait une image tout à fait adéquate à exprimer la virginité de la jeune fille, par sa fragilité certes, mais aussi par la promesse de fécondité qu'il représente.

L'angoisse de la virginité, dans la famille traditionnelle, est telle qu'en dehors de tous ces précautions et ces vertus éducatives, la mère recourt à la magie. Dans le souci de protéger la chasteté physique de sa fille et préserver sa dignité, elle n'hésite pas avant sa puberté à pratiquer certains rites qui sont communément utilisés et 
qui font appel à une sorte de mélange entre la magie et les croyances qu'on appelle «Sfah» ou «Tesfah» (fermeture invisible du vagin) ou, dans d'autres régions d'Algérie «R'bite» (nouée), ou la ferrure comme le décrit P. Bourdieu en détail dans son livre 'Esquisse d'une théorie de la pratique'. De cette façon, la fille sera protégée contre les risques de viol ainsi que contre ses propres emportements. Ainsi, nous pouvons dire que la thématique sexuelle et ses interdits constituent l'un des aspects essentiels de l'éducation traditionnelle de la fille.

\section{Conclusion:}

Telles sont en générale les caractéristiques de l'éducation de la fille au sein de la famille algérienne traditionnelle. Une éducation basée sur la conformité aux normes sociales, et qui porte souvent sur des aspects extérieurs, qui soumettent le comportement de la fille à son entourage, tels que l'obéissance, la politesse, la honte, le respect des autres, sans pour autant s'intéresser au caractéristiques individuelles comme l'indépendance, l'épanouissement et l'autonomie de l'enfant.

Ce regard peut nous pousser à en déduire que les individus du groupe familial traditionnel sont emprisonnés dans un mode contraignant, qu'ils sont des êtres malheureux à qui sont imposées des normes et des valeurs, qu'ils vivent comme contraintes et sont obligés de respecter. Pourtant une norme sociale n'est une contrainte que pour ceux qui ne s'y reconnaissent pas. De même que pour les 
valeurs, qui ne peuvent être contraignantes dans une société statique où il n'y a pas de changement dans les mentalités (contrairement a ce que nous vivons aujourd'hui). Les normes sociales à travers lesquelles se reproduit la famille traditionnelle ne sont pas vécues comme contraintes tant qu'elles correspondent aux représentations de la société. Ce modèle éducatif de la fille constitue un élément fondamental de leur personnalité de base et un moyen essentiel pour assurer l'identité sociale des sujets et garantir une meilleure adaptation à la société dans son ensemble. 


\section{Références:}

(1) Garnero. S, des relations précoces mères-enfant de la naissance à dixhuit mois en milieu culturel algérois, Mémoire de spécialisation en psychiatrie, Paris-Val- de marne, p95.

(2) Artificiel dans la famille traditionnelle veut dire le lait de vache ou de chèvre.

(3) M'rabet. F, 1979, Les femmes Algériennes, Paris, Cahiers libres, p 174.

(4) Lacoste Dujardin. C, , (1993), Des mères contre les femmes : maternité et patriarcat au Maghreb, Paris, L'harmattan, p 110.

(5) Lacoste Dujardin. C, 1976, Un village Algérien, Alger, SNED, pp 49-53.

(6) Zerdoumi. N, (1970), Enfant d'hier l'éducation de l'enfant dans le milieu traditionnelle Algérien, Paris, Maspéro, p $188 .$.

(7) Zerdoumi. N, op cit, p 186.

(8) Fanon. F, (1972), Sociologie d'une révolution, Paris, Maspéro, p 92.

(9) Ibid.

(10) Dufour. X L (sous direction), 1966, Vocabulaire de théologie biblique, Paris, les éditions du CERF, pp 1116-1119.

(11) Mernissi. F, 1983, Sexe, idéologie et Islam, Paris, édition Tiércé, p26. 
(12) Chebel. M, , (1993), L'imaginaire arabo-musulman, Paris, P.U.F, p322.

(13) Bourdieu. P, (1985), Sociologie de l'Algérie, Paris, P.U.F.

(14) Proverbe algérien.

(15) Zerdoumi. N, op cit, p190.

(16) Fanon. F, op cit, p 92.

(17) M'rabet. F, op cit, p 66.

(18) Lacoste Dujardin. C, (1993), op cit, p 87. 